\title{
Disfunção familiar em nonagenários e centenários: importância das condições de saúde e suporte social
}

\author{
Family dysfunction in nonagenarians and centenarians: \\ the importance of health conditions and social support
}

Ilva Inês Rigo (https://orcid.org/0000-0003-3073-5844) ${ }^{1}$

Ângelo José Gonçalves Bós (https://orcid.org/0000-0003-4901-3155) ${ }^{1}$

${ }^{1}$ Instituto de Geriatria e Gerontologia, Pontifícia Universidade Católica do Rio Grande do Sul. Av. Ipiranga 6681 Prédio $408^{\circ}$ andar sala 804 , Centro. 90619-900 Porto Alegre RS Brasil. ilvainesrigo@gmail.com

\begin{abstract}
The scope of this study is to evaluate factors related to family dysfunction (FD) among 227 randomly selected nonagenarians and centenarians in Porto Alegre, State of Rio Grande do Sul, who were visited and assessed in their homes. FD was evaluated by the "Family APGAR score," being considered with FD from 0 to 6, and without FD from 7 to 10 in this study. Sociodemographic and economic data, physical functionality, health self-perception, comorbidities, depressive symptoms, cognitive function, social support and interaction were evaluated. The mean APGAR score was 9.05 1.81, and FD occurred in $9.69 \%$ of the participants. Among the health conditions, the self-perception of health $(p=0.0003)$, the number of depressive symptoms $(p<0.0001)$, receiving visits $(p=0.0994)$, having recourse to help in case of illness ( $p=0.0090)$, and the need for help to administer medication ( $p=0.0602)$, were significantly related to FD. In the adjusted analysis, self-perception of health, the presence of depressive symptoms and the need for help in administering medication were associated with FD among nonagenarians and centenarians. These factors may influence the satisfaction of nonagenarians and centenarians with their family relationships. Key words Family relationships, Elderly individuals of 80 years old or more, Self-perception of health, Depressive symptoms
\end{abstract}

Resumo O presente estudo tem como objetivo avaliar os fatores relacionados à disfunção familiar (DF) entre 227 nonagenários e centenários identificados e avaliados no domicílio, aleatoriamente selecionados, em Porto Alegre, Rio Grande do Sul. A DF foi avaliada pelo instrumento "APGAR da família", sendo considerado, no presente estudo, de zero a seis com DF e de sete a dez sem DF. Foram avaliados, entre abril e novembro de 2016, dados sociodemográficos, econômicos, funcionalidade física, autopercepção de saúde, comorbidades, sintomas depressivos, função cognitiva, suporte e interação social. A média do APGAR foi de 9,05+1,81, a DF ocorreu em 9,69\% dos participantes. Foram relacionados à DF a autopercepção de saúde ( $p=0,0003)$, número de sintomas depressivos $(p<0,0001)$, ter ajuda em caso de doença $(p=0,0090)$ e necessidade de ajuda para administrar medicamentos $(p=0,0602)$. $\mathrm{Na}$ análise ajustada, foram independentemente associados à DF em nonagenários e centenários a autopercepção de saúde, a presença de sintomas depressivos e a necessidade de ajuda para administrar medicamentos. Conclui-se que esses fatores parecem interferir na satisfação do nonagenário ou centenário com suas relações familiares.

Palavras-chave Relações familiares, Idoso de 80 anos ou mais, Autopercepção de saúde, Sintomas depressivos 


\section{Introdução}

O envelhecimento é um processo multifatorial que ocorre ao longo de toda a vida. Do ponto de vista legal, o Estatuto do Idoso define como sendo idosa a pessoa com 60 anos ou mais de idade no Brasil'.

O processo de envelhecimento demográfico vem ocorrendo de forma muito rápida nos países em desenvolvimento. Entre os idosos, a faixa populacional dos com 80 anos ou mais, também chamados de longevos, é a que mais cresce ${ }^{2}$. Estima-se que, em 2050, essa faixa da população será de 13,3 milhões, representando $6,5 \%$ da população brasileira e 19,6\% da idosa ${ }^{3}$. Paschoal ${ }^{4}$ lembra que pessoas com idade acima da expectativa de vida apresentam maior chance de adoecimento e declínio da autonomia e da independência. Nas situações de perda da autonomia e da independência, o longevo necessita mobilizar fontes de auxílio.

A família é o espaço natural de cuidado aos indivíduos que a compõe. Com o idoso não é diferente $^{5}$. Entretanto, o peso desse processo de envelhecimento para as famílias, principalmente de países em desenvolvimento, tem sido muito grande. A família e outras instituições sociais informais assumem integralmente esse papel com pouco ou nenhum apoio do Estado. Sabe-se que famílias multigeracionais e com corresidência têm mais chance de prover cuidados, principalmente quando se trata de auxílio instrumental, nas atividades do dia a dia. Mas somente residir com alguém não implica em ser bem cuidado ${ }^{6}$. A funcionalidade familiar pode ser um fator relacionado a melhor qualidade de vida e saúde de longevos, pois espera-se que famílias funcionais sejam capazes de responder positivamente a situações críticas, como perda da funcionalidade e da autonomia do nonagenário e centenário ${ }^{7}$. Entretanto, pouco se tem estudado sobre a funcionalidade familiar nessa população, mesmo sendo os longevos o estrato populacional que mais cresce e que mais necessita de cuidado.

Um dos grupos que realiza o acompanhamento de longevos é o projeto Atenção Multiprofissional ao Longevo (AMPAL). O grupo realiza desde 2016 o acompanhamento domiciliar de pessoas com idade maior ou igual a 90 anos em diversos bairros de Porto Alegre ${ }^{8}$. A avaliação do AMPAL é baseada nas recomendações do Caderno de Atenção básica no 19 , intitulado Envelhecimento e Saúde da Pessoa Idoso ${ }^{7}$, que inclui a avaliação da funcionalidade familiar. Assim, o presente trabalho aborda os fatores relacionados com a disfunção familiar (DF) entre nonagenários e centenários do AMPAL.

\section{Método}

Trata-se de um estudo transversal analítico, com os dados coletados pelo AMPAL entre abril e novembro de 2016, com recursos do Fundo Municipal do Idoso de Porto Alegre. Os participantes incluídos foram nonagenários e centenários moradores de Porto Alegre identificados nas 17 regiões do Orçamento Participativo (OP) da cidade. O censo demográfico do IBGE de 2010 identificou 4.832 nonagenários e centenários residentes em Porto Alegre. O projeto AMPAL procurou, desta forma, identificar e avaliar $10 \%$ dessa população, ou seja em torno de 480 indivíduos. Utilizando a distribuição observada no censo, calculou-se o número de nonagenários e centenários a serem avaliados em cada OP, estabelecendo-se o número mínimo de 12 participantes em cada região. A partir dessa distribuição, foi sorteado um setor censitário para cada 12 nonagenários e centenários a serem avaliados em cada OP. Os setores censitários sorteados foram marcados em um mapa. Se em algum setor não pudesse ser identificado os 12 longevos, os setores dispostos ao redor deste seriam visitados, no sentido horário, a partir do primeiro setor à direita. Como o projeto foi afetado pela dificuldade da manutenção dos repasses do Fundo Municipal do Idoso, este logrou identificar e avaliar 245 nonagenários e centenários. A avaliação foi feita no domicílio do participante por uma dupla de profissionais da equipe, através de instrumento próprio, baseado nas recomendações do Caderno de Atenção Básica nº19, tema Envelhecimento e Saúde da Pessoa Idosa ${ }^{7}$. Os dados referentes ao Mini Exame do Estado Mental (MEEM), autopercepção de saúde, escala de depressão geriátrica e APGAR da família foram respondidos somente pelo nonagenário ou centenário. As demais questões puderam ser respondidas pelo cuidador. Entre os avaliados, 227 nonagenários e centenários tinham capacidade cognitiva para responder ao Apgar da Família. Nos casos em que o participante não tinha condições de responder, o entrevistador preencheu a opção "não interage com as pessoas" no questionário.

A funcionalidade familiar, no AMPAL, foi avaliada por meio do instrumento "Apgar da família", desenvolvido por Smilkstein ${ }^{9}$ e validado no Brasil por Duarte ${ }^{10}$. Esse instrumento foi o acrônimo das palavras "adaptability", "partner- 
ship", "growth", "affection" e "resolve", e avalia essas cinco dimensões da funcionalidade familiar, gerando um escore de 0 a 10. Esse escore foi estratificado em boa funcionalidade ( 7 a 10), moderada disfunção (5 e 6) e elevada disfunção (0 a 4). Para o presente estudo consideramos dois grupos: Apgar de 0 a 6, com DF; Apgar de 7 a 10 , sem DF. Foram incluídos na análise todos os nonagenários e centenários do AMPAL, que responderam ao questionário do Apgar da Família.

Também foram avaliados, como variáveis independentes, dados sociodemográficos, como escolaridade (saber ler e escrever e anos de estudo), situação domiciliar, estado conjugal, renda mensal (número de salários mínimos). A interação social foi descrita pela média de dias por mês que saiu de casa nos últimos seis meses, frequência que recebe visitas por semana, frequência que participa de atividades sociais por semana. O suporte social foi avaliado questionando ao idoso se alguém poderia ajudá-lo caso fique doente ou incapacitado, seguindo a orientação do Caderno de Atenção Básica no 19, do Ministério da Saúde ${ }^{8}$. A autopercepção de saúde (APS) foi respondida pelo próprio longevo, por meio de escolha simples das questões: ótima, boa, regular, má, péssima ou não soube responder, no caso do longevo não ter condições para tal. A mensuração da condição cognitiva foi realizada por meio do MEEM, criado por Folstein et al. ${ }^{11}$ e validado no Brasil por Bertolucci et al. ${ }^{12}$

A funcionalidade física foi avaliada através de sete atividades cotidianas: caminhar 400 metros ou quatro quadras, subir 10 degraus ou um lance de escadas, levantar ou carregar objetos de cinco quilos, levantar-se da cadeira sem usar as mãos, agachar-se e levantar-se para pegar um objeto no chão, levantar os braços acima da cabeça, agarrar objetos firmemente com as mãos; e cinco atividades básicas da vida diária: transferir-se para uma cama ou cadeira, tomar banho sozinho, se vestir sozinho, alimentar-se sozinho e usar o banheiro, conforme a abordagem de Morsch et al. ${ }^{13}$. As atividades foram classificadas pelo participante por grau de dificuldade (fácil, mais ou menos fácil, difícil, não consegue). As atividades foram pontuadas de acordo com o desempenho: fácil ( 5 pontos), mais ou menos fácil (3 pontos), difícil (1 ponto) e não consegue, zero, totalizando, no máximo 35 pontos para atividades cotidianas e 25 para básicas. O percentual de funcionalidade física foi obtido considerando-se o percentual de capacidade executiva sobre a pontuação total das atividades cotidianas e básicas. Um nonagenário ou centenário totalmente funcional seria classificado com $100 \%$ de desempenho funcional e básico. A funcionalidade física foi classificada de acordo com o percentual de desempenho nas duas atividades. A triagem para depressão geriátrica foi realizada com a Geriatric Depression Scale de cinco itens (GDS-5), validada por Almeida ${ }^{14}$. Conforme a autora, a suspeita de depressão ocorre quando houver pontuação igual ou maior a 2 .

A análise foi realizada através do programa EpiInfo versão 7. Foi comparada a distribuição dos fatores sociodemográficos e clínicos com a funcionalidade familiar (com e sem DF). As médias da idade, renda mensal familiar, número de doenças crônicas (comorbidades), sintomas depressivos, pontuação no MEEM, frequência semanal de participação em atividades sociais e número de dias mensais que sai de casa foram calculados para cada nível de DF e as diferenças testadas pelo T de Student. A distribuição dos demais fatores foram calculados para cada nível de DF e testadas pelo Qui-Quadrado, considerado o nível de significância estatística de $5 \%(p<0,05)$. As variáveis com estatisticamente significativas nas análises descritivas foram incluídas em um modelo de regressão logística, para as chances de ter DF, inicialmente simples e após ajustada entre si. Em cada variável incluída no modelo uma categoria foi selecionada como referência. A regressão logística atribui o valor de 1 para essa categoria. As com maiores chances de ter DF apresentam razões de chance maiores que 1 , e as com menor chance, valores menores do que 1 . Variáveis não significativas no modelo ajustado inicial foram excluídas no modelo final, restando somente os fatores significativos.

\section{Aspectos éticos}

O Projeto AMPAL foi aprovado pelo Comitê de Ética em pesquisa da PUC-RS. Todos os participantes já assinaram um Termo de Consentimento Livre e Esclarecido autorizando a realização da pesquisa e o contato telefônico.

\section{Resultados}

A Tabela 1 mostra a distribuição das características sociodemográficas dos nonagenários e centenários do projeto AMPAL de acordo com a presença ou não de DF, ou seja, Apgar da família menor ou igual a 6. Foram incluídos na análise 227 nonagenários e centenários, dos quais, 22 (9,69\%) apresentaram DF. A média do Apgar da família foi de $9,05 \pm 1,81$, sendo significati- 
Tabela 1. Características sociodemográficas dos nonagenários com e sem DF, n=227 nonagenários e centenários, Porto Alegre, Rio Grande do Sul, 2016.

\begin{tabular}{|c|c|c|c|c|}
\hline & Sem DF & Com DF & Total & $\mathbf{p}$ \\
\hline APGAR (média \pm DP) & $9,56 \pm 0,79$ & $4,27 \pm 1,69$ & $9,05 \pm 1,81$ & $<0,0001$ \\
\hline Idade (média $\pm \mathrm{DP})$ & $92,32 \pm 3,58$ & $91,82 \pm 2,82$ & $92,27 \pm 3,51$ & 0,5273 \\
\hline \multicolumn{5}{|l|}{ Sexo $n(\%)$} \\
\hline Feminino & $151(90,96)$ & $15(9,04)$ & $166(73,13)$ & \multirow[t]{2}{*}{0,5819} \\
\hline Masculino & $54(88,52)$ & $7(11,48)$ & $61(26,87)$ & \\
\hline \multicolumn{5}{|l|}{ Cor $\mathrm{n}(\%)$} \\
\hline Branca & $166(89,25)$ & $20(10,75)$ & $186(81,94)$ & \multirow[t]{2}{*}{0,3826} \\
\hline Não branca & $39(95,12)$ & $2(4,88)$ & $41(18,06)$ & \\
\hline \multicolumn{5}{|l|}{ Estado Conjugal n(\%) } \\
\hline Casado(a) & $44(93,62)$ & $3(6,38)$ & $47(20,70)$ & \multirow[t]{4}{*}{0,6272} \\
\hline Divorciado(a) & $6(100,00)$ & $0(0,00)$ & $6(2,64)$ & \\
\hline Solteiro(a) & $19(86,36)$ & $3(13,64)$ & $22(9,69)$ & \\
\hline Viúvo(a) & $136(89,47)$ & $16(10,53)$ & $152(66,96)$ & \\
\hline \multicolumn{5}{|l|}{ Sabe ler e escrever $n(\%)$} \\
\hline Não & $24(96,00)$ & $1(4,00)$ & $25(11,01)$ & \multirow[t]{2}{*}{0,4814} \\
\hline Sim & $181(89,60)$ & $21(10,40)$ & $202(88,99)$ & \\
\hline Anos de estudo(média $\pm D P$ ) & $6,00 \pm 4,49$ & $7,36 \pm 4,04$ & $6,14 \pm 4,46$ & 0,1747 \\
\hline Renda mensal $(\mathrm{SM})($ média $\pm \mathrm{DP})$ & $5,96 \pm 11,03$ & $4,50 \pm 6,75$ & $5,82 \pm 10,69$ & $0,2111^{*}$ \\
\hline \multicolumn{5}{|l|}{ Com quem mora $\mathrm{n}(\%)$} \\
\hline Cuidador & $16(84,21)$ & $3(15,79)$ & $19(8,37)$ & \multirow[t]{4}{*}{0,6335} \\
\hline Familiar & $156(90,70)$ & $16(9,30)$ & $172(75,77)$ & \\
\hline Sozinho & $33(91,67)$ & $3(8,33)$ & $36(15,86)$ & \\
\hline Total n(\%) & $205(90,31)$ & $22(9,69)$ & $227(100,00)$ & \\
\hline
\end{tabular}

$\mathrm{DP}=$ desvio padrão; $\mathrm{SM}=$ salários mínimos; * Teste não paramétrico de Kruskal-Wallis $\mathrm{H}$.

Fonte: Elaborado pelos autores.

va a diferença entre os participantes com e sem DF $(\mathrm{p}<0,0001)$. Observa-se que a média etária dos participantes foi de 92,27 anos (DP $\pm 3,51$, sendo semelhante entre os grupos com e sem DF $(p=0,5273)$. A participante mais longeva tinha 108 anos na data da entrevista. Com relação ao sexo, $73 \%$ dos participantes era do sexo feminino, a cor da pele autodeclarada com mais frequência foi a branca (82\%), 67\% eram viúvos, $89 \%$ sabiam ler e escrever e, destes, o tempo médio de estudo foi de 6 anos, a renda mensal foi de 5,8土10,69 salários mínimos, 76\% residiam com familiar. Não houve diferenças e associações significativas entre as características sociodemográficas e a disfunção familiar. Apesar disso, apresentaram maior frequência de DF os homens, brancos, solteiros, alfabetizados, com mais anos de estudo, menor renda e que residiam com o cuidador formal (não familiar).

A Tabela 2 se refere às condições de saúde dos nonagenários do AMPAL com e sem DF. A APS mais frequente foi ótima ou boa $(63 \%)$ e a DF ocorreu mais entre os nonagenários que consideravam sua saúde má ou péssima (31,58\%); sendo a associação entre níveis de APS e DF estatisticamente significativa $(\mathrm{p}=0,0003)$. Quanto à presença de sintomas depressivos, os nonagenários relataram em média $1,49 \pm 1,13$ sintomas. Os participantes com DF relataram em média

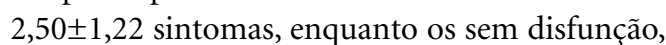
$1,38 \pm 1,06$ sintomas, sendo essa diferença estatisticamente significativa $(\mathrm{p}<0,0001)$. Referente aos escores no MEEM, a pontuação geral foi de $21,01 \pm 6,23$. A pontuação foi bastante semelhante entre os grupos com e sem DF $(20,18 \pm 7,21$ e $21,10 \pm 6,13$, respectivamente; $p=0,5115$. O número de comorbidades foi em média de $4,48 \pm 2,22$, bastante semelhante entre os grupos com e sem DF $(4,68 \pm 3,15$ e $4,46 \pm 2,10$, respectivamente) $(\mathrm{p}=0,9052)$. A maior parte $(86,78 \%)$ dos participantes relatou ter recebido algum atendimento de saúde nos últimos 6 meses. A frequência de DF foi semelhante entre os grupos que receberam ou não atendimento $(\mathrm{p}=1,00)$. 
No que se refere à participação e interação social, a Tabela 3 mostra que $78,41 \%$ recebiam visitas. Entre os nonagenários que não recebiam, $16,33 \%$ relataram DF, enquanto que $7,87 \%$ dos que recebiam visitas referiram DF. Essa diferença foi indicativa de significância $(\mathrm{p}=0,0994)$. Quando questionados sobre a participação em atividades sociais, como grupos de idosos, a participação foi de $0,45 \pm 1,18$ dias por semana. No geral, $81,06 \%$ disseram não participar dessas atividades. A presença de DF foi mais comum entre os que não participavam de atividades so- ciais $(10,87 \%)$ do que entre os que participavam $(4,65 \% ; p=0,2660)$. Em relação a sair de casa, os nonagenários do AMPAL relataram sair em média $10,37 \pm 11,15$ dias por mês nos últimos 6 meses $(p=0,6571)$. Quase todos $(99,12 \%)$ os sujeitos de pesquisa afirmaram ter com quem contar em caso de doença. Somente dois nonagenários relataram não ter ajuda e justamente estes tiveram escore de Apgar da família compatível com DF. A diferença dos participantes com ou sem apoio em caso de doença foi significativa entre os grupos com e sem DF ( $\mathrm{p}=0,0090)$.

Tabela 2. Condições de saúde dos nonagenários com e sem DF, n=227 nonagenários e centenários, Porto Alegre/ RS, 2016.

\begin{tabular}{|c|c|c|c|c|}
\hline & Sem DF n(\%) & Com DF n(\%) & Total $\mathbf{n}(\%)$ & $\mathbf{p}$ \\
\hline \multicolumn{5}{|l|}{ Autopercepção de saúde } \\
\hline Ótima ou boa & $136(95,10)$ & $7(4,90)$ & $143(63,00)$ & 0,0003 \\
\hline Regular & $55(87,30)$ & $8(12,70)$ & $63(27,75)$ & \\
\hline Má ou péssima & $13(68,42)$ & $6(31,58)$ & $19(8,37)$ & \\
\hline NSR & $1(50,00)$ & $1(50,00)$ & $2(0,88)$ & \\
\hline Número de Comorbidades & $4,46 \pm 2,10$ & $4,68 \pm 3,15$ & $4,48 \pm 2,22$ & 0,9052 \\
\hline GDS-5 & $1,38 \pm 1,06$ & $2,50 \pm 1,22$ & $1,49 \pm 1,13$ & $<0,0001$ \\
\hline MEEM & $21,10 \pm 6,13$ & $20,18 \pm 7,21$ & $21,01 \pm 6,23$ & 0,5115 \\
\hline \multicolumn{5}{|c|}{ Atendimentos de saúde nos últimos 6 meses } \\
\hline Não & $27(90,00)$ & $3(10,00)$ & $30(13,22)$ & 1,0000 \\
\hline Sim & $178(90,36)$ & $19(9,64)$ & $197(86,78)$ & \\
\hline Total & $205(90,31)$ & $22(9,69)$ & $227(100,00)$ & \\
\hline
\end{tabular}

GDS-5: Pontuação na Escala de Depressão Geriátrica de 5 itens; MEEM: Mini Exame do Estado Mental.

Fonte: Elaborado pelos autores.

Tabela 3. Interação e suporte social dos nonagenários com e sem DF, $\mathrm{n}=227$ nonagenários e centenários, Porto Alegre/RS, 2016.

\begin{tabular}{|c|c|c|c|c|}
\hline & Sem DF & Com DF & Total & $\mathbf{p}$ \\
\hline \multicolumn{5}{|l|}{ Recebe visitas $\mathrm{n}(\%)$} \\
\hline Não & $41(83,67)$ & $8(16,33)$ & $49(21,59)$ & 0,0994 \\
\hline $\operatorname{Sim}$ & $164(92,13)$ & $14(7,87)$ & $178(78,41)$ & \\
\hline \multicolumn{5}{|l|}{ Participação em atividades Sociais n(\%) } \\
\hline Não & $164(89,13)$ & $20(10,87)$ & $184(81,06)$ & 0,2660 \\
\hline $\operatorname{Sim}$ & $41(95,35)$ & $2(4,65)$ & $43(18,94)$ & \\
\hline Atividades sociais (por semana) média $\pm D P$ & $0,46 \pm 1,18$ & $0,41 \pm 1,22$ & $0,45 \pm 1,18$ & 0,8389 \\
\hline Frequência mensal de saída de casa média $\pm D P$ & $10,47 \pm 11,18$ & $9,36 \pm 11,12$ & $10,37 \pm 11,15$ & 0,6571 \\
\hline \multicolumn{5}{|c|}{ Possui alguém que possa lhe ajudar em caso de doença n(\%) } \\
\hline Não & $0(0,00)$ & $2(100,00)$ & $2(0,88)$ & $0,0090^{*}$ \\
\hline Sim & $205(91,11)$ & $20(8,89)$ & $225(99,12)$ & \\
\hline Total n(\%) & $205(90,31)$ & $22(9,69)$ & $227(100,00)$ & \\
\hline
\end{tabular}

* teste exato de Fischer; DP: desvio padrão. 
Ao abordarmos a funcionalidade física, na Tabela 4 , vemos que os participantes mantinham um bom desempenho em atividades básicas, como tomar banho, vestir-se, alimentar-se, usar o banheiro, de $79,03 \pm 28,42 \%$. O desempenho básico dos nonagenários sem DF foi maior $(79,53 \pm 27,85)$ que os com DF $(74,36 \pm 33,69 ; p=0,4189)$. Em relação ao desempenho funcional, como caminhar 400 metros, subir um lance de escada, carregar 5 quilos, levantar da cadeira sem usar as mãos, os participantes mantinham $58,65 \pm 26,14 \%$ dessas capacidades. O desempenho básico foi menor entre os nonagenários com DF $(53,78 \pm 26,63$; $\mathrm{p}=0,4189)$. Ao questionarmos sobre a capacidade de sair da cama sozinho, a maior parte $(85,02 \%)$ era capaz. A DF foi mais frequente entre os incapazes de sair da cama sozinho $(11,76 \%$; $\mathrm{p}=0,7519)$. Quando foram abordadas atividades mais complexas, como fazer compras e preparar refeições, verificamos um aumento da incapacidade se comparadas com atividades mais simples, já que $53,30 \%$ afirmaram não conseguir fazer compras e $36,12 \%$ não eram capazes de preparar refeições. Os grupos com e sem DF foram semelhantes, tanto para fazer compras $(\mathrm{p}=0,9022)$, como preparar refeições $(0,9803)$, embora a frequência de DF tenha sido maior nos com menor desempenho. Mais da metade $(53,74)$ dos nonagenários relataram não necessitar de ajuda para controlar e administrar seus medicamentos. A DF foi mais frequente entre os que não necessitavam de ajuda com seus medicamentos $(13,11 \%)$ ao comparada com os que necessitavam de ajuda (5,71), essa diferença foi indicativa de significância $(p=0,0602)$.

A Tabela 5 mostra a regressão logística simples e ajustada para as variáveis que foram significativas ou indicativas de significância: APS, presença de sintomas depressivos, receber ou não visitas, ajuda para administrar medicamentos e para o caso de adoecimento agudo. A variável relacionada à ajuda em caso de doença não pode ser incluída pois apenas dois participantes relataram não ter ajuda e ambos com DF, tornando a regressão logística inválida. Na análise simples, a APS ótima ou boa mostrou ser um fator de proteção para a DF, em relação aos nonagenários e centenários com pior APS, a razão de chance calculada pela regressão logística foi de 0,11 , representando $89 \%$ menos chance de ter DF (1-0,11), sendo esta associação significativa $(\mathrm{p}=0,0005)$. Ajustando para as demais variáveis, a significância estatística diminui ( $\mathrm{p}=0,0259)$. Observamos que a presença de um sintoma depressivo a mais aumentava em $129 \%$ a chance de ter DF $(p<0,0001)$. Esse fator seguiu significativo após o ajuste pelas demais variáveis $(\mathrm{p}=0,0005)$. Receber visitas, que era indicativo de significância na análise simples $(\mathrm{p}=0,0827)$, na análise ajustada deixa de ser significativo ( $p=0,2284$ ). A necessidade de ajuda com medicamentos que era indicativo de significância na análise simples $(\mathrm{p}=0,0673)$, passou a ser significativo na ajustada ( $\mathrm{p}=0,0146)$.

Tabela 4. Funcionalidade física dos participantes com e sem DF, $\mathrm{n}=227$ nonagenários e centenários, Porto Alegre/RS, 2016.

\begin{tabular}{|c|c|c|c|c|}
\hline & Sem DF & Com DF & Total & $\mathbf{p}$ \\
\hline Desempenho Básico (\%médio $\pm \mathrm{DP})$ & $79,53 \pm 27,85$ & $74,36 \pm 33,69$ & $79,03 \pm 28,42$ & 0,4189 \\
\hline Desempenho Funcional (\%médio $\pm \mathrm{DP})$ & $59,18 \pm 26,10$ & $53,78 \pm 26,63$ & $58,65 \pm 26,14$ & 0,3581 \\
\hline \multicolumn{5}{|l|}{ Sai da cama sozinho $\mathrm{n}(\%)$} \\
\hline Sim & $175(90,67)$ & $18(9,33)$ & $193(85,02)$ & 0,7519 \\
\hline Não & $30(88,24)$ & $4(11,76)$ & $34(14,98)$ & \\
\hline \multicolumn{5}{|l|}{ Faz compras sozinho $\mathrm{n}(\%)$} \\
\hline Sim & $96(90,57)$ & $10(9,43)$ & $106(46,70)$ & 0,9022 \\
\hline Não & $109(90,08)$ & $12(9,92)$ & $121(53,30)$ & \\
\hline \multicolumn{5}{|l|}{ Prepara refeições n(\%) } \\
\hline Sim & $131(90,34)$ & $14(9,66)$ & $145(63,88)$ & 0,9803 \\
\hline Não & $74(90,24)$ & $8(9,76)$ & $82(36,12)$ & \\
\hline \multicolumn{5}{|l|}{ Necessita ajuda com medicamentos $\mathrm{n}(\%)$} \\
\hline $\operatorname{Sim}$ & $106(86,89)$ & $16(13,11)$ & $122(53,74)$ & 0,0602 \\
\hline Não & $99(94,29)$ & $6(5,71)$ & $105(46,26)$ & \\
\hline Total n(\%) & $205(90,31)$ & $22(9,69)$ & $227(100,00)$ & \\
\hline
\end{tabular}

Fonte: Elaborado pelos autores. 
Tabela 5. Regressão logística simples e ajustada dos fatores relacionados com a DF entre nonagenários do Projeto AMPAL, n=227 nonagenários e centenários, Porto Alegre/RS, 2016.

\begin{tabular}{lcccccc}
\hline & \multicolumn{3}{c}{ Simples } & \multicolumn{3}{c}{ Ajustada } \\
\cline { 2 - 7 } & RC & IC 95\% & $\mathbf{p}$ & RC & IC 95\% & p \\
\hline Autopercepção se saúde (ref. Má ou péssima) & & & & & \\
$\quad$ Ótima ou boa & 0,11 & $0,03-0,38$ & 0,0005 & 0,19 & $0,04-0,88$ & 0,0259 \\
$\quad$ Regular & 0,32 & $0,09-1,07$ & 0,0634 & 0,38 & $0,07-1,46$ & 0,1404 \\
$\quad$ NSR & 2,17 & $0,12-40,81$ & 0,6057 & 6,42 & $0,19-217,7$ & 0,3008 \\
Sintomas depressivos & 2,30 & $1,54-3,42$ & $<0,0001$ & 2,26 & $1,42-3,58$ & 0,0005 \\
$\quad$ Recebe visitas (ref. Não) & & & & & \\
$\quad$ Sim & 0,44 & $0,17-1,11$ & 0,0827 & 0,49 & $0,15-1,56$ & 0,2284 \\
$\quad$ Necessita de ajuda com medicamentos (ref. Não) & & & & & \\
$\quad$ Sim & 0,40 & $0,15-1,07$ & 0,0673 & 0,21 & $0,06-0,74$ & 0,0146 \\
\hline
\end{tabular}

RC: Razão de Chances; IC95\%: Intervalo de Confiança com 95\% de confiabilidade.

Fonte: Elaborado pelos autores.

\section{Discussão}

No presente trabalho, a boa funcionalidade familiar foi apresentada pela maior parte dos nonagenários e centenários entrevistados, semelhante ao que ocorreu no estudo de Vera et al. ${ }^{15}$ com longevos de 80 anos ou mais da comunidade. À exceção deste estudo, o nível do Apgar dos participantes do AMPAL foi melhor que em outros estudos, inclusive realizados com idosos mais jovens, com 60 anos ou mais ${ }^{16-21}$. A média do Apgar foi também maior que a de um estudo chinês $(6,9 \pm 2,3)$ realizado, igualmente, com nonagenários e centenários ${ }^{22}$, em que prevaleceu a DF (52,2\%). É possível verificar que a frequência de DF entre os participantes do AMPAL foi menor que a apresentada em diversos estudos, tanto com longevos quanto com idosos.

Quando consideradas as variáveis sociodemográficas, não ocorreu diferença significativa entre os grupos com e sem DF. Destaca-se na literatura, o sexo feminino como protetor para DF, como apresentado por De Oliveira et al. ${ }^{17}$, em estudo com idosos institucionalizados, o mesmo observado no presente estudo, onde homens tinham maior frequência de DF. Por outro lado, um estudo realizado com adultos portugueses, que teve média etária de 52 anos, associou o sexo feminino à $\mathrm{DF}^{23}$. Esses diferentes achados podem ser explicados pelas faixas etárias diferentes nos artigos. De acordo com o papel social e familiar da mulher, como mãe e provedora de cuidados ao longo da vida, espera-se que ela tenha melhor apoio familiar e consequente menor frequência de DF que entre os homens.
Em relação ao estado civil e a renda, Prazeres e Santiago ${ }^{23}$ observaram, em pessoas idosas portuguesas, similaridades com o presente estudo, usando o Apgar como instrumento. A maior frequência de DF também ocorreu entre os idosos portugueses não casados e com baixa renda. Porém, os autores observaram maior frequência de DF entre os que moravam sós, oposto do que o apresentado nos participantes do AMPAL, em que a DF foi mais frequente nos que residiam com cuidador ou com familiar. Ao avaliarmos a escolaridade, observamos que os participantes do AMPAL com maior instrução tinham maior frequência de DF, sendo esse um relato não observado na literatura estudada. Acreditamos que isso se deva por terem maior crítica de sua situação familiar em relação aos com menor escolaridade.

Ao levarmos em consideração as condições de saúde dos nonagenários e centenários de nossa pesquisa, observamos que a APS, predominou a ótima ou, semelhante ao que ocorreu em outro estudo com idosos jovens usuários de restaurantes populares ${ }^{24}$. No presente estudo, a APS parece estar intimamente relacionada com a funcionalidade familiar. Os participantes com APS ruim ou péssima relataram maior frequência de DF. Esse achado corrobora com outros estudos como o de Takeda, Sasagawa \& Mori $^{19}$ em japoneses e Vera et al. ${ }^{15}$ em brasileiros. A APS parece estar relacionada com a percepção de DF entre os participantes. Da mesma forma, os sintomas depressivos também parecem influenciar a funcionalidade familiar. Em nosso estudo, essa variável foi significativamente maior entre os nonagenários e centenários com DF. O mesmo ocorreu em ou- 
tros estudos nacionais e internacionais ${ }^{17-19}$. Como não foram encontrados estudos que utilizassem a GDS 5, não foi possível comparar a média de sintomas depressivos com outros estudos.

O número de comorbidades, o acesso a serviços de saúde e o desempenho no MEEM não foram relacionados significativamente com a DF em nossa amostra, sendo bastante semelhantes entre os grupos. A média MEEM dos sujeitos do AMPAL foi maior que de outros estudos com nonagenários e centenários chineses ${ }^{22,25}$. De Oliveira et al..$^{17} \mathrm{e}$ Vera et al. ${ }^{15}$ também não verificaram relação da saúde cognitiva com a funcionalidade familiar. Com relação ao número de comorbidades, Vera et al. ${ }^{15}$ não realizaram a contabilização do total por participante, mas relacionaram a funcionalidade familiar com osteoporose, dado incomum na literatura, enquanto que as demais patologias não tiveram associação significativa. Nosso estudo não verificou relação entre o número de comorbidades e a DF. Parece ser mais importante o julgamento que o nonagenário/ centenário faz de sua saúde que o número de doenças diagnosticadas. A busca por serviços de saúde nos últimos seis meses também não diferiu entre os grupos com e sem DF. Vera et al. ${ }^{15}$ verificaram essa diferença, mas essa variável não se manteve significativa na regressão logística daquele estudo, observado a sua dependência a outros fatores. Prazeres e Santiago ${ }^{23}$, com sujeitos adultos maiores de 18 anos, estabeleceram uma relação com o maior número de comorbidades (embora não estatisticamente significativa) e de necessidades de saúde não atendidas com a DF. É possível que a melhor funcionalidade familiar facilite também o acesso e $o$ atendimento às necessidades de saúde.

Ao avaliarmos a interação e o apoio social, receber visitas foi indicativo de significância, com maior frequência de DF entre os que não recebiam visitas. Ter com que contar em caso de doença foi significativo, sendo os sujeitos que não tinham essa disponibilidade os que apresentaram DF. A falta de apoio social talvez repercuta na percepção da DF, embora no presente estudo, ter com quem contar em caso de adoecimento não tenha significância estatística após o controle por outras variáveis. Lambotte et al. ${ }^{26}$ corroboram com essa afirmação, já que o cuidado informal, oferecido por familiares, amigos e vizinhos, foi o mais frequentemente acessado pelos idosos. Esses achados se assemelham a um estudo japonês, em que o pior índice de interação social foi associado com baixo índice de funcionalidade familiar $(\mathrm{RC}=2,80 ; \mathrm{p}<0,001)$, mesmo após ajuste por outras variáveis ${ }^{19}$. Martins et al. ${ }^{20}$ observaram maior frequência de abuso e maus-tratos entre idosos que sentiam solidão. Outro estudo, da Turquia, relacionou o suporte social com melhor qualidade de vida ${ }^{27}$.

A funcionalidade física não diferiu entre os sujeitos do AMPAL com e sem DF. Verificamos maior desempenho em atividades simples, como transferir-se da cama para uma cadeira e pior nas que exigem mais habilidades cognitivas, como preparar refeições e fazer compras. A dificuldade nestas atividades ocorreu apesar do bom desempenho no MEEM da nossa amostra. Somente a questão de necessitar de ajuda para controlar e tomar seus medicamentos teve diferença significativa, especificamente os participantes que não necessitavam de ajuda relataram mais frequentemente DF. Isso pode ser possível por que a percepção de necessidade de ajuda do nonagenário/ centenário deve equilibrar os momentos em que não está satisfeito com sua família. Talvez o idoso acabe se submetendo a situações que lhe desagradam devido a essa necessidade de cuidado da família. Essa relação de dependência foi demonstrada por Sun et al. ${ }^{28}$, que verificaram melhor adesão ao tratamento medicamentos entre pacientes esquizofrênicos com melhor funcionalidade familiar. Não foram encontrados outros estudos que demonstrassem a relação entre dependência para o uso de medicamentos e funcionalidade familiar. Em nosso estudo, essa variável se manteve significativa na regressão logística, mesmo após o ajuste pelas demais, demonstrando ser um fator independente.

\section{Conclusão}

A análise ajustada demonstrou relação significativa entre funcionalidade familiar e APS, presença de sintomas depressivos e necessidade de ajuda para o uso de medicamentos, mesmo após o controle por outras variáveis.

Concluímos que a APS, a presença de sintomas depressivos e a dependência para administrar medicamentos parecem interferir na satisfação do nonagenário ou centenário com suas relações familiares. Novamente, sendo a família a principal fonte de cuidados ao longo da vida, em especial no final dela, faz-se necessário estimular o cultivo de relações saudáveis, tanto para quem cuida, quanto para quem é cuidado. Fatores sociodemográficos, desempenho funcional e apoio social não foram preditores significativos de funcionalidade familiar. 


\section{Colaboradores}

II Rigo responsabilizou-se pela elaboração do projeto, coleta e análise dos dados e redação do artigo. A Bós orientou todas as etapas do trabalho e participou da revisão e redação do projeto e do artigo.

\section{Financiamento}

O presente trabalho foi realizado com apoio da Coordenação de Aperfeiçoamento de Pessoal de Nível Superior - Brasil (CAPES), código de financiamento 001. Bolsa de Mestrado concedida a Ilva Inês Rigo.

\section{Referências}

1. Brasil. Estatuto do Idoso. $3^{\mathrm{a}}$ ed. $2^{\mathrm{a}}$ reimpr. Brasília: Ministério da Saúde; 2013.

2. Camacho NCA, Morche KR, Muller AL, Bós AJG. Por que nonagenários não se tornam centenários no Brasil? AMRIGS 2018; 62(1):55-59.

3. Camarano AA, Kanso S. Envelhecimento da população brasileira: uma contribuição demográfica. In: Freitas EV, Py L. Tratado de geriatria e gerontologia. Rio de Janeiro: Guanabara Koogan; 2016. p.141-164.

4. Paschoal SMP. Qualidade de vida na velhice. In: Freitas EV, Py L. Tratado de geriatria e gerontologia. Rio de Janeiro: Guanabara Koogan; 2016. p. 184-194.

5. Lemos ND, Medeiros SL. Suporte social ao idoso dependente. In: Freitas EV, Py L. Tratado de geriatria e gerontologia. Rio de Janeiro: Guanabara Koogan; 2016. p.2211-2218.

6. Debert GG, Simões JA. Envelhecimento e velhice na família contemporânea. In: Freitas EV, Py L. Tratado de geriatria e gerontologia. Rio de Janeiro: Guanabara Koogan; 2016. p.1571-1579.

7. Brasil. Ministério da Saúde, Secretaria de Atenção à Saúde, Departamento de Atenção Básica (MS/SAS/ $\mathrm{DAB})$. Envelhecimento e saúde da pessoa idosa. Brasília: MS/SAS/DAB; 2006.

8. Pontifícia Universidade Católica do Rio Grande do Sul (PUC-RS). Projeto Longevidade [Internet]. [acessado 2017 out 11]. Disponível em: http://longevidade. pucrs.br/quem-somos/

9. Smilkstein G. The Family APGAR: A proposal for family function test and its use by physicians. J fam pract 1978; 6(6):1231-1239.

10. Duarte YAO. Família: rede de suporte ou fator estressor. A ótica de idosos e cuidadores familiares [tese]. São Paulo (SP): Universidade de São Paulo; 2001.

11. Folstein MF, Folstein SE, McHugh PR. Mini-mental state: a practical method for grading the cognitive state of patients for the clinician. J Psychiatr Res 1975; 12(3):189-198.

12. Bertolucci PH, Brucki SM, Campacci SR, Juliano Y.O Mini Exame do Estado Mental em uma população geral: impacto da escolaridade. Arq Neuropsiquiatr 1994; 52(1):1-7.

13. Morsch P, Pereira G N, Navarro JHDN, Trevisan MD, Lopes DGC, Bós AJG. Características clínicas e sociais determinantes para o idoso sair de casa. Cad Saude Publica 2015; 31(5):1025-1034.

14. Almeida MSC. Efetividade da escala de depressão geriátrica de cinco itens em população idosa da comunidade [tese]. Porto Alegre: PUCRS; 2010.

15. Vera I, Lucchese R, Nakatani AYK, Pagotto V, Montefusco SRA, Sadoyama G. Funcionalidade familiar em longevos residentes em domicílio. Rev Bras Enferm 2015; 68(1):68-75.

16. Lu C, Yuan L, Lin W, Zhou Y, Pan S. Depression and resilience mediates the effect of family function on quality of life of the elderly. Arch Gerontol Geriatr 2017; 71:34-42.

17. Oliveira SC, Pavarini SCI, Orlandi FS, Mendiondo MSZ. Family functionality: a study of Brazilian institutionalized elderly individuals. Arch Gerontol Geriatr 2014; 58(1):170-176. 
18. Almeida Souza R, Costa GD, Yamashita CH, Amendola F, Gaspar JC, Martins Alvarenga MR, Oliveira MAC. Funcionalidade familiar de idosos com sintomas depressivos. Rev Esc Enferm USP 2014; 48(3):469-476.

19. Takeda S, Sasagawa Y, Mori M. Personal characteristics associated with individual degree of family function in residents of Rumoi City, Hokkaido. J Gen Fam Med 2017; 18(6):372-377.

20. Martins R, Neto M, Andrade A, Albuquerque C. Abuse and maltreatment in the elderly. Atención Primaria 2014; 46:206-209.

21. Hai S, Wang H, Cao L, Liu P, Zhou J, Yang Y, Dong B. Association between sarcopenia with lifestyle and family function among community-dwelling Chinese aged 60 years and older. BMC geriatrics 2017; 17(187):1-7.

22. Wang B, He P, Dong B. Association between family functioning and cognitive impairment among Chinese nonagenarians/centenarians. Geriatr Gerontol Int 2015; 15:1135-1142.

23. Prazeres F, Santiago L. Relationship between health -related quality of life, perceived family support and unmet health needs in adult patients with multimorbidity attending primary care in Portugal: a multicentre cross-sectional study. Health Qual Life Outcomes 2016; 14:1-12.

24. Gomes MFS, Pereira SCL, Abreu MNS. Fatores associados à autopercepção de saúde dos idosos usuários dos restaurantes populares de Belo Horizonte. Cien Saude Colet 2018; 23(11):4007-4019.

25. Deng J, Hu J, Wu W, Dong B, Wu H. Subjective well -being, social support, and age-related functioning among the very old in China. Int J Geriatr Psychiatry 2010; 25(7):697-703.
26. Lambotte D, De Donder L, Van Regenmortel S, Fret B, Dury S, Smetcoren AS, Dierckx E, De Witte N, Verté D, Kardol MJM, D-SCOPE Consortium. Frailty differences in older adults' use of informal and formal care. Arch Gerontol Geriatr 2018; 79: 69-77.

27. Unalan D, Gocer S, Basturk M, Baydur H, Ozturk A. Coincidence of low social support and high depressive score on quality of life in elderly. Eur Geriatr Med 2015; 6(4):319-324.

28. Sun, Y, Wang M, Zhou Y, Wang L, Zhang H, Lv Y, Li G. The mediating effect of family function and medication adherence between symptoms and mental disability among Chinese patients with schizophrenia: a cross-sectional study. Psychol Health Med 2018:1-11.

Artigo apresentado em 03/01/2019

Aprovado em 27/08/2019

Versão final apresentada em 29/08/2019

Editores-chefes: Romeu Gomes, Antônio Augusto Moura da Silva 